\title{
Glioblastoma multiforme presenting as cryptogenic intracerebral hemorrhage
}

\author{
Jose M. Soto, MD (D), Kristopher A. Lyon, MD (D) and Ethan A. Benardete, MD, PhD (D) \\ Department of Neurosurgery, Baylor Scott and White Health, Temple, Texas
}

\begin{abstract}
We report a case of glioblastoma multiforme that presented as a large, spontaneous intracerebral hemorrhage (ICH). A 52-yearold woman developed headaches associated with photophobia and nausea. Computed tomography scan revealed a large ICH in the right temporal lobe. Further imaging, including magnetic resonance imaging and catheter-based arteriography, did not demonstrate an underlying lesion. She had 2-month follow-up imaging that was consistent with resolution of her hematoma and, again, no evidence of an associated mass. Six months after the initial ICH, she presented with worsening headaches. Magnetic resonance imaging of her brain at that time clearly demonstrated a large enhancing mass in the area of prior hemorrhage. Following tumor resection, histological diagnosis indicated glioblastoma multiforme. This case highlights the benefit of neurosurgical follow-up for patients with cryptogenic ICH and maintaining a high index of suspicion for brain tumor.
\end{abstract}

KEYWORDS Cryptogenic hemorrhage; glioblastoma multiforme; intracerebral hemorrhage; primary brain tumor

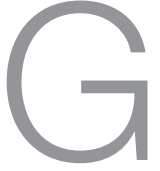

lioblastoma multiforme (GBM), the most common primary brain tumor in adults, typically presents with gradually progressive neurologic symptoms. ${ }^{1}$ In rare cases, however, the patient may present with rapid neurologic decline due to intracerebral hemorrhage $(\mathrm{ICH})$. In patients presenting with spontaneous $\mathrm{ICH}$, the incidence of previously undiagnosed brain tumors has been estimated to be $0.6 \% .^{2}$ It can be difficult to discern underlying neoplastic pathology on initial imaging, and delayed diagnosis is common. In this report, we discuss the presentation and imaging of a rare case of GBM presenting as a large, spontaneous $\mathrm{ICH}$.

\section{CASE PRESENTATION}

A 52-year-old woman with known discoid lupus and diabetes mellitus presented to her primary care physician after a few days of headache, nausea, photophobia, and gait instability. She was diagnosed with a viral syndrome, but her symptoms continued for several weeks. The patient was sent for a computed tomography (CT) brain scan, which revealed an intraparenchymal hemorrhage in the right temporal lobe $(4.5 \times 4 \times 3.1 \mathrm{~cm})$ (Figure 1). There was also a $3-\mathrm{mm}$ mid- line shift, mild vasogenic edema, and effacement of the right lateral ventricle.

In the emergency department, the patient had mild weakness in her left upper and lower extremity but otherwise a normal neurologic examination. She denied recent head trauma or anticoagulant use. CT angiography of the brain did not demonstrate an underlying vascular lesion. Magnetic resonance imaging (MRI) of the brain with and without gadolinium contrast, likewise, did not reveal an underlying mass (Figure 2). The patient underwent catheter-based cerebral angiography, which did not show an underlying vascular abnormality. She improved neurologically while in the hospital and was discharged home on levetiracetam for seizure prophylaxis.

After 4 weeks, the patient returned to the emergency department complaining of an intermittent metallic taste associated with periods of altered awareness. She was diagnosed with complex partial seizures. MRI of the brain without contrast demonstrated partial resolution of her hematoma but no evidence of an underlying mass. She was discharged on a higher dose of levetiracetam and had no further symptoms. The patient maintained regular follow-up in the neurosurgery clinic, and 2 months later, repeat MRI brain with and without contrast showed resolution of her

Corresponding author: Jose M. Soto, MD, Department of Neurosurgery, Baylor Scott and White Health, 2401 S. 31 Street, Temple, TX 76508 (e-mail: Jose.Soto@bswhealth.org)

Received April 13, 2018; Revised June 29, 2018; Accepted July 6, 2018. 


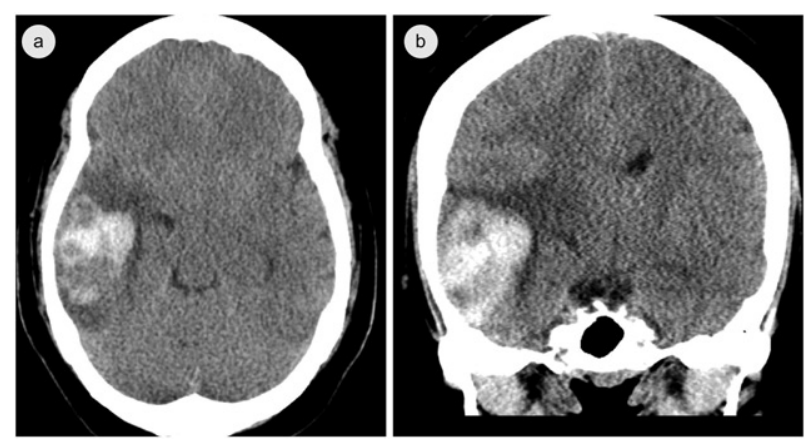

Figure 1. Noncontrast head CT from the initial admission. (a) Axial and (b) coronal views show a large hemorrhage in the right temporal lobe.
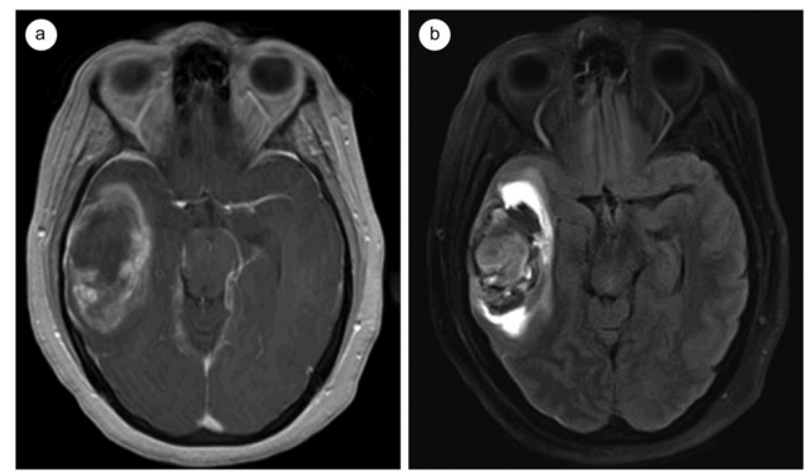

Figure 2. MRI of the brain from the initial admission. (a) T1-weighted image after gadolinium administration shows no evidence of an underlying mass. The bright surrounding signal is subacute hemorrhage. (b) The fluid-attenuated inversion recovery image shows the subacute blood products in the right temporal lobe with surrounding edema.
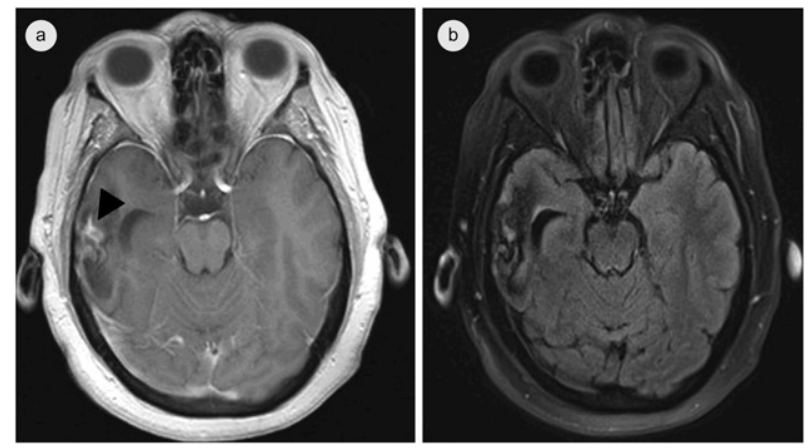

Figure 3. Axial MRI of the brain 2 months after initial presentation. (a) T1weighted image after gadolinium administration shows no clear evidence of tumor. The small area of enhancement (arrowhead) was interpreted as reactive gliosis. (b) The fluid-attenuated inversion recovery image shows no significant edema or mass.

hematoma, and no other mass was identified by the radiologist (Figure 3).

Six months after her initial presentation, the patient experienced worsening headaches and returned to the emergency department. Noncontrast CT of the brain revealed a prominent mass and edema in the right temporal lobe. MRI of the brain now clearly demonstrated a large, enhancing mass $(5.2 \times 3.5 \times 3.2 \mathrm{~cm})$ in the area of prior hemorrhage
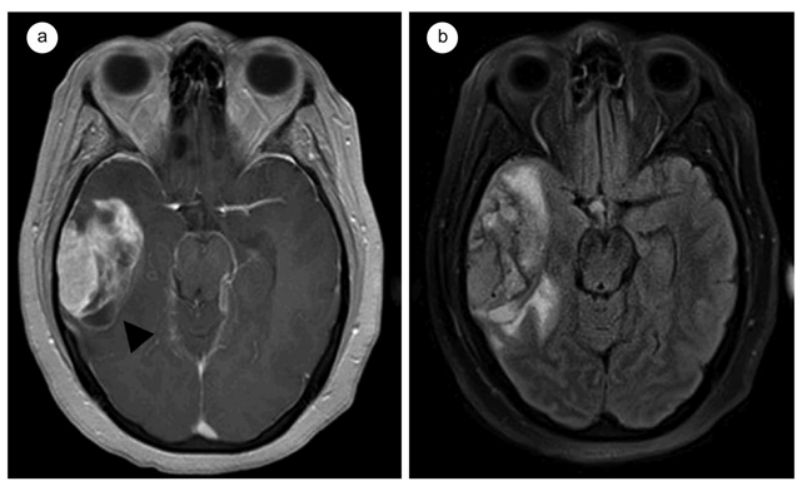

Figure 4. Axial MRI of the brain obtained 6 months after initial presentation. (a) There is a large enhancing mass (arrowhead) on the T1-weighted image after gadolinium contrast administration in the right temporal lobe. (b) On the fluid-attenuated inversion recovery image, there is a heterogeneous mass with surrounding edema in the right temporal lobe.

(Figure 4). The patient underwent craniotomy and gross total resection of the enhancing mass 201 days after her initial presentation. Pathological evaluation demonstrated a GBM with significant invasion of the adjacent dura. Molecular pathology showed isocitrate dehydrogenase wildtype, $\mathrm{O}^{6}$ methylguanine-DNA methyltransferase (MGMT) methylation, chromosome 1 polysomy with deletion of $1 \mathrm{p} 36 / 1 \mathrm{p} 25$, and chromosome 19 polysomy with intact 19q. The patient is currently being treated with temozolomide after a course of standard external beam radiation.

\section{DISCUSSION}

In adults, spontaneous ICH is most frequently associated with chronic hypertension. ${ }^{3}$ Primary brain tumors, such as GBM, can rarely manifest as spontaneous ICH with a incidence between $0.54 \%$ and $3.4 \% .{ }^{4}$ Tumor-associated hemorrhage is likely due to direct vascular invasion as well as the development of abnormal, fragile vessels supplying the tumor. ${ }^{5}$ On initial imaging, it can be difficult to discern an underlying mass. The tumor may be compressed by the hematoma, and the border between blood and tumor may be obscured. In tumor-associated ICH, perilesional edema is a frequent finding. ${ }^{6}$

In patients with GBM who present with spontaneous $\mathrm{ICH}$, diagnosis is often delayed, with a median time to diagnosis of 60 days. $^{7}$ Histological examination of the hematoma may allow for early detection of tumor. However, hematoma sampling is frequently not done since several clinical trials have failed to support surgical evacuation of $\mathrm{ICH}$, particularly in patients who remain stable or are improving after initial hemorrhage. ${ }^{8}$ Newer surgical techniques, such as minimally invasive clot evacuation, could play a role in obtaining tissue for diagnosis in cases of cryptogenic ICH. ${ }^{9}$ In most cases, close monitoring of patients who have had a spontaneous intracerebral hemorrhage of no apparent cause is indicated. ${ }^{10}$ 
ORCID

Jose M. Soto (D) http://orcid.org/0000-0001-8609-6901

Kristopher A. Lyon (iD http://orcid.org/0000-0003-3095-2697

Ethan A. Benardete (iD http://orcid.org/0000-0001-7341-3744

1. Wen PY, Kesari S. Malignant gliomas in adults. $N$ Engl J Med. 2008;359(5):492-507. doi: 10.1056/NEJMra0708126.

2. Wakai S, Yamakawa K, Manaka S, Takakura K. Spontaneous intracranial hemorrhage caused by brain tumor: Its incidence and clinical significance. Neurosurgery. 1982;10(4):437-444. doi: 10.1227/00006123198204000-00004.

3. Qureshi AI, Tuhrim S, Broderick JP, Batjer HH, Hondo H, Hanley DF. Spontaneous intracerebral hemorrhage. $N$ Engl J Med. 2001; 344(19):1450-1460. doi: 10.1056/NEJM200105103441907.

4. Iwama T, Ohkuma A, Miwa Y, et al. Brain tumors manifesting as intracranial hemorrhage. Neurol Med Chir (Tokyo). 1992;32(3): 130-135. doi: 10.2176/nmc.32.130.

5. Choi G, Park DH, Kang SH, Chung YG. Glioma mimicking a hypertensive intracerebral hemorrhage. J Korean Neurosurg Soc. 2013;54(2): 125-127. doi: 10.3340/jkns.2013.54.2.125.
6. Schrader B, Barth H, Lang EW, et al. Spontaneous intracranial haematomas caused by neoplasms. Acta Neurochir (Wien). 2000;142(9):979-985.

7. Joseph DM, O'Neill AH, Chandra RV, Lai LT. Glioblastoma presenting as spontaneous intracranial haemorrhage: Case report and review of the literature. J Clin Neurosci. 2017;40:1-405. doi: 10.1016/j.jocn.2016.12.046.

8. Mendelow AD, Gregson BA, Fernandes HM, et al. Early surgery versus initial conservative treatment in patients with spontaneous supratentorial intracerebral haematomas in the International Surgical Trial in Intracerebral Haemorrhage (STICH): A randomised trial. Lancet. 2005;365(9457):387-397. doi: 10.1016/S01406736(05)70233-6.

9. $\mathrm{Xu} \mathrm{X}$, Chen $\mathrm{X}, \mathrm{Li} \mathrm{F}$, et al. Effectiveness of endoscopic surgery for supratentorial hypertensive intracerebral hemorrhage: A comparison with craniotomy. J Neurosurg. 2018;128(2):553-559. doi: 10.3171/ 2016.10.JNS161589.

10. Thaler PB, Li JY, Isakov Y, Black KS, Schulder M, Demopoulos A. Normal or non-diagnostic neuroimaging studies prior to the detection of malignant primary brain tumors. J Clin Neurosci. 2012;19(3): 411-414. doi: 10.1016/j.jocn.2011.09.002. 